\title{
Anchoring and Localizing Single Metal Atoms for Better Catalysis
}

\author{
Jingyue (Jimmy) Liu, Jia Xu, Yang Lou, Yafeng Cai and Xu Li
}

Department of Physics, Arizona State University, Tempe, Arizona, USA

Aberration-corrected scanning transmission electron microscopy (STEM), especially the high-angle annular dark-field (HAADF) imaging mode, has played a critical role in the rapid advances in catalysis by supported single metal atoms [1-5]. The sub-angstrom resolution and the atomic number sensitivity of the STEM-HAADF imaging technique make it possible not only to routinely examine the dispersion of supported single metal atoms in practical catalysts but also to obtain the coordinate information of the adsorbed metal atoms with respect to the surface structure of a crystalline support [6]. Such atomic scale information on the coordinates of the supported single metal atoms has been directly used to establish models for DFT calculations of the catalytic cycles of single-atom catalysts (SACs) [2]. The STEMHAADF imaging technique has proved to be indispensable for optimizing synthesis protocols of SACs, studying the stability of SACs during catalytic reactions, and probing the nature of catalytically active centers in SACs [6].

A critical challenge to practical applications of SACs is the stability of the supported single metal atoms during catalytic reactions, especially at high reaction temperatures. When a single metal atom occupies a cation vacancy position of a metal oxide support its strong interaction with the surrounding oxygen ions can form chemical bonds with the support and thus resist to sintering during a catalytic reaction (Figure 1a). New anchoring sites are needed when the number of metal atoms exceeds that of the surface cation vacancy sites on a metal oxide support. Surface steps, various types of surface or near surface defects, or impurities become important anchoring sites to stabilize single metal atoms. Because of their high surface area and plenty of surface defects two dimensional, polycrystalline metal oxide supports can accommodate high levels of metal loading while still maintain the characteristics of the isolated metal atoms (Figure 1b). Activated carbons are frequently used for liquid phase catalysis due to their resistance to acid or base attack. The interaction between single metal atoms and the edges of graphene (graphite) sheets or other types of defects within the activated carbons becomes critical and such interactions can be utilized to stabilize single metal atoms (Figure 1c) and modify the electronic structure of the anchored metal atoms. When the support crystals do not interact strongly with the targeted metal and do not contain many surface defects the isolated single metal atoms cannot be stabilized. For example, $\mathrm{Pt}$ atoms can only decorate the edges of $2 \mathrm{D} \mathrm{MoS}_{2}$ layers but cannot be anchored on the surfaces of the $\mathrm{MoS}_{2}$ crystal layers (Figure 1d).

During a catalytic reaction over a SAC, the stability of the supported single metal atoms strongly depends on the gas or liquid environment as well as the reaction temperature. For example, for metal oxides supported SACs the anchored single metal atoms usually are stable under oxidation environment, even at elevated temperatures. Under reducing environments (e.g, $\mathrm{H}_{2}$ or $\mathrm{CO}$ ), however, the anchoring ligands (e.g., oxygen ions) can be modified and thus destabilize the anchored single metal atoms. Such a strong dependence of the SAC stability on the reaction environment poses a grand challenge to broad practical applications of SACs. Engineering and functionalization of support surfaces become imperative for further advancing the SAC-based catalytic technologies. New strategies such as construction of nanostructured diffusion barriers, isolation of individual single metal atoms, and creation of more surface cation vacancies will be discussed [7]. 


\section{References:}

[1] J Liu, ChemCatChem 3 (2011), p.934.

[2] B Qiao et al, Nat Chem 3 (2011), p.634.

[3] X-F Yang et al, Acc Chem Res 45 (2013), p.1740.

[4] J Liu, ACS Catal 7 (2017), p.34.

[5] B C Gates et al, Catal Sci Technol 7 (2017), p.4259.

[6] J Liu, Chinese J Catal 38 (2017), p.1460.

[7] This work was funded by NSF under CHE-1465057. We acknowledge use of facilities in the John M. Cowley Center for High Resolution Electron Microscopy at Arizona State University.
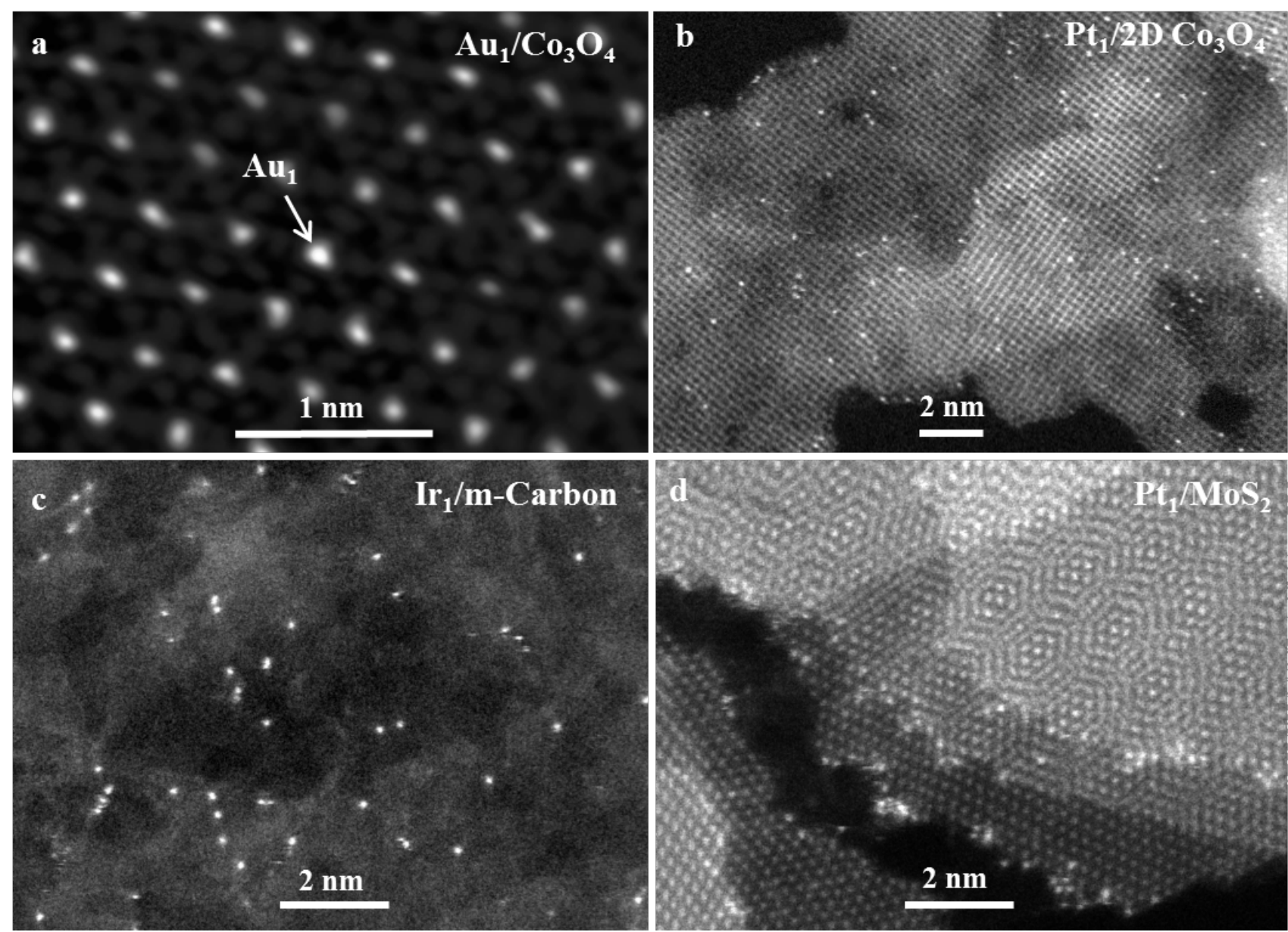

Figure 1. Atomic resolution STEM-HAADF images of (a) a single $\mathrm{Au}_{1}$ atom anchored at the surface $\mathrm{Co}$ cation vacancy position on the $\mathrm{Co}_{3} \mathrm{O}_{4}$ support, (b) isolated $\mathrm{Pt}_{1}$ atoms anchored at surface steps, grain boundaries, or point defects on the surfaces of the two-dimensional $\mathrm{Co}_{3} \mathrm{O}_{4}$ support, (c) single $\mathrm{Ir}_{1}$ atoms anchored at the edges and defect sites of graphene nano-flakes in a highly meso-porous hollow carbon support, and (d) $\mathrm{Pt}_{1}$ atoms decorating the edges of the monolayers of $\mathrm{MoS}_{2}$ support material. The stabilization of the single metal atoms in the single-atom catalysts strongly depends on defect anchoring (vacancies, steps, edges, etc.). Clustering of metal atoms may occur if the anchoring strength is not strong enough or if the anchoring sites are modified during a catalytic reaction, especially at elevated temperatures and/or under reducing environment. 